\title{
防撓矩形板の撓に就いて
}

\section{正貝工学士 樋口道之助}

Abstract.

Rectangular Plate with Reinforcements.

By Michinosuke Higuchi, Kogakushi, Member.

This investigation considers the problem of deflections and strength of the rectangular plate with reinforcements under general distribution of loading, and under the boundary conditions in which one pair of opposite edges normal to reinforcements are simply supported, the other pair of edges are elastically supported, by reinforcements. And all reinforcements have rigidity against twist as well as against deflection, and those are built in with brackets which have elastic constants for angular displacements.

We express the influences of reinforcements on the rectangular plate in Dirac's impulse func tion $\delta\left(x, x_{i}\right)$ and $\delta^{\prime}\left(x, x_{i}\right)$, and we apply the double or single Fourier Transformation to solve the equation of equilibrium of the rectangular plate under the above boundary conditions.

\section{§1．緒霄}

防撓矩形板の撓及強度については，已に数回に渉り発表してきたものであるが，以前の論文において周辺の条 件には一定の限度があり, 自由に境界条件を取り得なかつた。即ち以前の論文に执いては， $x=0 ， x=a$ の辺は 弾性固定とし $y=0, b$ の辺は単純支持とした。周辺における撓はすべて許されなかつた。本論文に扎いては上記 条件を大幅に緩和すると共に，防撓材の端部も実際の場合を考慮して肘板固着の場合を考えることとする。

本論文における境界条件及附加条件を列記 すれば，下記の如きものである。

1) $y=0, b$ の辺は以前と同じく単純支持 とするも防撓材の端部には肘板を附してあ るので単純支持ではなく弾性固定とする。

2） $x=0, a$ の辺においては撓 $w \neq 0$ であ つて, この辺にも防撓材を附加し, 彷つて 挨机剛性の存在による角変位曲存在する。

3）防撓材はすべて撓に対する剛性を有す ると共に㧖れに対する剛性も有すること以 前の論文と同様である。

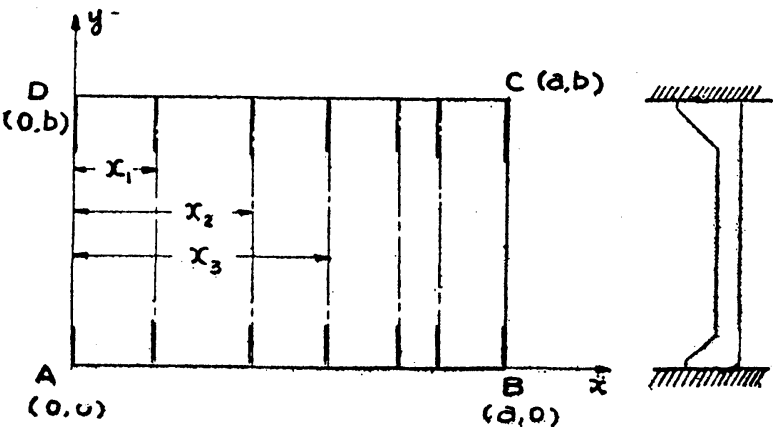
斯様な境界条件並びに附加条件を满足する

解を求めるに以前の論文においては, $w=\sum_{m, n} a_{9 n n} \sin \frac{m \pi x}{a} \sin \frac{n \pi y}{b}$ と置き係数 $a_{m n}$ を求めるに無限マトリック ス方程式を解き，解を得たのであるが；本論文においては，フーリエ変換を使用して解を求めることにする。こ の方法に依れば, 解法は割に簡易化され有限元の連立方程式を解けばよいことになる。勿論数学的な意味は全く 同一であるが，境界条件，附加条件が自然に入る便があるのである。

本論文においても，集中荷重及び集中モーメントを表わすに衝撃函数 $\delta, \delta^{\prime}$ を利用した。

\section{$\S 2$ 記号}

$w$ ： 矩形板の撓 
$D:$ 矩形板の撓岡度 $=\frac{1}{12} \frac{E h^{3}}{1-v^{2}}$

$E: \quad "$ " "のヤング係数

$E_{i}: i$ 番目の防撓材のヤング係数

$I_{i}:$ " " " " " " "の断面二次モーメント

$G_{i}: " \quad "$ " の剪断弾性俰数

$K_{i}:$ " " " " の㨝りの断面係数

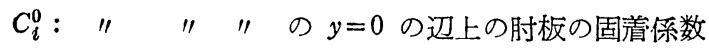

$C_{i}^{b}: "$ " の $y=b$ の " "

$p ：$ 荷重

$\nu:$ ポアソン比

$h:$ 矩形板の厚さ

$$
\begin{aligned}
& \lambda=a / b \quad B=\lambda^{4} n^{4} \sum_{\alpha} \frac{1}{\left(\alpha^{2}+\lambda^{2} n^{2}\right)^{2}} \\
& \gamma_{i}=\frac{2 E_{i} I_{i}}{D a} \quad a^{0} m n=\frac{4}{a b D} \frac{1}{\left\{\left(\frac{m \pi}{a}\right)^{2}+\left(\frac{n \pi}{b}\right)^{2}\right\}^{2}} \int_{0}^{a} \int_{0}^{b} p \sin \frac{m \pi \xi}{a} \sin \frac{n \pi \eta}{b} d \xi d \eta \\
& \theta_{i}=\frac{2 G_{i} K_{i}}{D a} \quad p_{c}: \text { 防撶材上の集中荷重 } \\
& K=\frac{\pi^{2} E I}{4 D a} \quad p_{c}^{o}=\int_{0}^{b} p_{c} \sin \frac{m \pi \eta}{b} d \eta \\
& \bar{C}_{0}^{0}=\frac{\pi C_{0}^{0}}{E I a} \quad F=\sum_{\alpha} a_{\alpha_{\alpha n}^{0} \alpha} \\
& \bar{C}_{b}^{0}=\frac{\pi C_{0}^{b}}{E I a} \quad G=\frac{1}{\lambda^{2} n^{2}} \sum_{\alpha} a_{\alpha n}^{0}\left\{\alpha^{3}+(2-v) \alpha \lambda^{2} n^{2}\right\} \\
& A=\lambda^{2} n^{2} \sum_{\alpha} \frac{\alpha^{2}}{\left(\alpha^{2}+\lambda^{2} n^{2}\right)^{2}} \quad G_{1}=\frac{1}{\lambda^{2} n^{2}} \sum_{\alpha} a_{\alpha n}^{0}\left(\alpha^{2}+2 \lambda^{2} n^{2}\right) \alpha \\
& \delta\left(x, x_{i}\right), \delta^{\prime}\left(x, x_{i}\right): \text { Dirac } 9 \text { 第 } 0 \text { 位及第 } 1 \text { 位の衝撃函数 } \\
& M_{1}=\sum_{\beta} \frac{K A}{K A \lambda^{2} \beta^{2}+(A+B)^{2}} \\
& M_{2}=\sum_{\beta} \frac{K A(-1)^{\beta}}{K A \lambda^{2} \beta^{2}+(A+B)^{2}} \\
& M=\sum_{\beta} \frac{A}{K A \lambda^{2} \beta^{2}+(A+B)^{2}} \quad \beta \text { は奇数 } \\
& N_{1}=\sum_{\beta} H \lambda \beta \\
& N_{2}=\sum_{\beta} H(-1)^{\beta} \lambda \beta \\
& H=\frac{A G_{1}+B F}{K A \lambda^{2} H^{2}+(A+B)^{2}} \\
& N_{1}{ }^{\prime}=\sum_{\beta} H \lambda \beta \quad \beta \text { は奇数, } M_{1}{ }^{\prime}=\sum_{\beta} \frac{K A}{K A \lambda^{2} \beta^{2}+(A+B)^{2}} \quad \beta \text { は奇数 }
\end{aligned}
$$

猶 $x=x_{i}, y=b$ 等の点に拈淤る函数の值を表わすに, $w_{i}^{b},\left(\frac{\partial w}{\partial x}\right)_{0}^{0}$ の如く右下に $x$ 座漂, 右上に $y$ 座標の指 数を附す。

\section{$\S 3$ 境 界 条 件}

緒言に述べた如き境界条件並びに附加条件を式により表現すれば，次の如きものとなる。

$$
y=0, b \text { に打いて } w=0, \frac{\partial^{2} w}{\partial x^{2}}=\frac{\partial^{2} w}{\partial y^{2}}=0
$$


但し防撓材の端部を凡て除く

$$
\begin{aligned}
& x=0, a \text { に於いて } \\
& E_{0} I_{0}\left(\frac{\partial^{4} w}{\partial y^{4}}\right)_{0}=D\left\{\frac{\partial^{3} w}{\partial x^{3}}+(2-v)-\frac{\partial^{3} w}{\partial x \partial y^{2}}\right\}_{x=0} \\
& E_{a} I_{a}\left(\frac{\partial^{4} w}{\partial y^{4}}\right)_{a}=D\left\{\frac{\partial^{3} w}{\partial x^{3}}+(2-v) \frac{\partial^{3} w}{\partial x \partial y^{2}}\right\}_{x=a} \\
& -G_{0} K_{0}\left(\frac{\partial^{3} w}{\partial x \partial y^{2}}\right)_{0}=D\left(\frac{\partial^{2} w}{\partial x^{2}}+v \frac{\partial^{2} w}{\partial y^{2}}\right)_{0} \\
& -G_{a} K_{a}\left(\frac{\partial^{3} w}{\partial x \partial y^{2}}\right)_{a}=D\left(\frac{\partial^{2} w}{\partial x^{2}}+v \frac{\partial^{2} w}{\partial y^{2}}\right)_{a} \\
& x=0, \quad y=0 \text { において } E_{0} I_{0}\left(\frac{\partial^{2} w}{\partial y^{2}}\right)_{0}^{0}=-C_{0}^{0}\left(-\frac{\partial w}{\partial y}\right)_{0}^{0} \\
& x=0, \quad y=b \text { において } E_{0} I_{0}\left(\frac{\partial^{2} w}{\partial y^{2}}\right)_{0}^{b}=-C_{0}^{b}\left(\frac{\partial w}{\partial y}\right)_{0}^{b} \\
& x=a, \quad y=0 \text { において } E_{a} I_{a}\left(\frac{\partial^{2} w}{\partial y^{2}}\right)_{a}^{0}=-C_{a}^{0}\left(\frac{\partial w}{\partial y}\right)_{a} \\
& x=a, \quad y=b \text { において } E_{a} I_{a}\left(\frac{\partial^{2} w}{\partial y^{2}}\right)_{a}^{b}=-C_{a}^{b}\left(\frac{\partial w}{\partial y}\right)_{a}^{b}
\end{aligned}
$$

更に $x=x_{i}$ なる位置にある防撓材の端部においては,

$$
\begin{aligned}
& x=x_{i}, y=0 \text { に打いて } E_{i} I_{i}\left(\frac{\partial^{2} w}{\partial y^{2}}\right)_{i}^{0}=-C_{i}^{0}\left(\frac{\partial w}{\partial y}\right)_{i}^{0} \\
& x=x_{i}, y=b \text { において } E_{i} I_{i}\left(\frac{\partial^{2} w}{\partial y^{2}}\right)_{i}^{b}=-C_{i}^{b}\left(\frac{\partial w}{\partial y}\right)_{i}^{b}
\end{aligned}
$$

以上は防撓材の存在する場合の条件式であるが, 若し $x=0, a$ 又は $x_{i}$ の位置の中で何れか防撓材が無く, 単 飞肘板のみが附加されている場合には（6 ） (11)式の中これに相等するものは下記の式と取換光ねばならない。

$$
\begin{array}{ll}
x=0, y=0 \text { に於いて } D\left(\frac{\partial^{2} w}{\partial y^{2}}+v \frac{\partial^{2} w}{\partial x^{2}}\right)_{0}^{0}=-C_{0}^{0}\left(\frac{\partial w}{\partial y}\right)_{0}^{0} \\
x=0, y=b \text { に於いて } D\left(\frac{\partial^{2} w}{\partial y^{2}}+v \frac{\partial^{2} w}{\partial x^{2}}\right)_{0}^{b}=-C_{0}^{b}\left(\frac{\partial w}{\partial y}\right)_{0}^{b} \\
x=a, y=0 \text { に於いて } D\left(\frac{\partial^{2} w}{\partial y^{2}}+v \frac{\partial^{2} w}{\partial x^{2}}\right)_{a}^{0}=-C_{a}^{0}\left(\frac{\partial w}{\partial y}\right)_{a}^{0} \\
x=a, y=b \text { に於いて } D\left(\frac{\partial^{2} w}{\partial y^{2}}+v \frac{\partial^{2} w}{\partial x^{2}}\right)_{a}^{b}=-C_{a}^{b}\left(\frac{\partial w}{\partial y}\right)_{a}^{b} \\
x=x_{i}, y=0 \text { に於いて } D\left(\frac{\partial^{2} w}{\partial y^{2}}+v \frac{\partial^{2} w}{\partial x^{2}}\right)_{i}^{0}=-C_{i}^{0}\left(\frac{\partial w}{\partial y}\right)_{i}^{0} \\
x=x_{i}, y=b \text { に於いて } D\left(\frac{\partial^{2} w}{\partial y^{2}}+v \frac{\partial^{2} w}{\partial x^{2}}\right)_{i}^{b}=-C_{i .}^{b}\left(\frac{\partial w}{\partial y}\right)_{i}^{b}
\end{array}
$$

\section{$\S 4$ 一 般 理 論}

緒言に述べた如き防撓材の附加された場合の矩形板の平衡方程式は以前の論文と同じく下記の如く表わされる

$$
D \Delta \Delta w=p-\sum_{i}\left\{E_{i} I_{i}\left(\frac{\partial^{4} w}{\partial y^{4}}\right)_{x=x_{i}} \delta\left(x, x_{i}\right)+G_{i} K_{i}\left(\frac{\partial^{3} w}{\partial x \partial y^{2}}\right)_{x=x_{i}} \delta^{\prime}\left(x, x_{i}\right)\right\}
$$

我々は(18)式を(1)〜(11)又はこれ等に代るべき(12)〜(17)の条件下に解かねばならない。 (18)式の両辺に下記の如く, 变数 $x, y$ に関する二重フーリエ変換を適用すれば,

$$
\begin{aligned}
& \int_{0}^{a} \int_{0}^{b} D \Delta \Delta w \sin \frac{m \pi x}{a} \sin \frac{n \pi y}{b} d x d y=\int_{0}^{a} \int_{0}^{b} p \sin \frac{m \pi x}{a} \sin \frac{n \pi y}{b} d x d y \\
& -\sum_{i} \int_{0}^{a} \int_{0}^{b}\left\{E_{i} I_{i}\left(\frac{\partial^{4} w}{\partial y^{4}}\right)_{x=x_{i}} \delta\left(x, x_{i}\right)+G_{i} K_{i}\left(\frac{\partial^{3} w}{\partial x \partial y^{2}}\right)_{x=x_{i}} \delta^{\prime}\left(x, x_{i}\right)\right\} \sin \frac{m \pi x}{a} \sin \frac{n \pi y}{b} \\
& d x d y
\end{aligned}
$$

而してフーリエ変換の理論に依り 


$$
\begin{aligned}
\int_{0}^{a} \int_{0}^{b} \Delta & \Delta w \sin \frac{m \pi x}{a} \sin \frac{n \pi y}{-b} d x d y=\left\{\left(\frac{m \pi}{a}\right)^{2}+\left(\frac{n \pi}{b}\right)^{2}\right\}^{2} \int_{0}^{a} \int_{0}^{b} w \sin \frac{m \pi x}{a} \sin \frac{n \pi y}{b} d x d y \\
& +\left\{\left(\frac{m \pi}{a}\right)^{3}+2\left(\frac{m \pi}{a}\right)\left(\begin{array}{c}
n \pi \\
b
\end{array}\right)^{2}\right\}\left\{\int_{0}^{b} w_{0} \sin \frac{n \pi y}{b} d y-(-1)^{m} \int_{0}^{b} w_{a} \sin \frac{n \pi y}{b} d y\right\} \\
& +\left\{\left(\frac{n \pi}{b}\right)^{3}+2\left(\frac{n \pi}{b}\right)\left(\frac{m \pi}{a}\right)^{2}\right\}\left\{\int_{0}^{a} w^{0} \sin \frac{m \pi x}{a} d x-(-1)^{n} \int_{0}^{a} w^{b} \sin \frac{m \pi x}{a} d x\right\} \\
& +\left(\frac{m \pi}{a}\right)\left\{\int_{0}^{b}\left(\frac{\partial^{2} w}{\partial x^{2}}\right)_{c} \sin \frac{n \pi y}{b} d y-(-1)^{m} \int_{0}^{b}\left(\frac{\partial^{2} w}{\partial x^{2}}\right)_{a} \sin \frac{n \pi y}{b} d y\right\} \\
& +\left(\frac{n \pi}{b}\right)\left\{\int_{b}^{a}\left(\frac{\partial^{2} w}{\partial y^{2}}\right)^{0} \sin \frac{m \pi x}{a} d x-(-1)^{n} \int_{0}^{a}\left(\frac{\partial^{2} w}{\partial y^{2}}\right)^{b} \sin \frac{m \pi x}{a} d x\right\} \\
& +2\left(\frac{m \pi}{a}\right)\left(\frac{n \pi}{b}\right)\left\{w_{0}^{0}-(-1)^{m} w_{0}^{o}-(-1)^{n} w_{b}^{0}+(-1)^{m+n} w_{a}^{b}\right\}
\end{aligned}
$$

又衝撃函数 $\delta\left(x, x_{i}\right)$ 及 $\delta^{\prime}\left(x, x_{i}\right)$ の性質を考慮して, 下記の二式を゙得ることが出来る。

$$
\begin{aligned}
& E_{i} I_{i} \int_{0}^{a} \int_{0}^{b}\left(\frac{\partial^{4} w}{\partial y^{4}}\right)_{x_{i}} \delta\left(x, x_{i}\right) \sin \frac{m \pi x}{a} \sin \frac{n \pi y}{b} d x d y \\
& =E_{i} I_{i}^{\Gamma}\left(\frac{n \pi}{b}\right)^{4} \int_{0}^{b} w_{x_{i}} \sin \frac{n \pi y}{b} d y+\left(\frac{n \pi}{b}\right)\left\{\left(\frac{\partial^{2} w}{\partial y^{2}}\right)_{i}^{o}-(-1)^{\prime} \frac{\partial^{2} w}{\partial y^{2}}\right)_{i}^{b}\left[\left\{\sin \frac{m \pi x_{i}}{a}\right.\right. \\
& E_{i} K_{i} \int_{0}^{a} \int_{0}^{b}\left(\frac{\partial^{3} w}{\partial x \partial y^{2}}\right)_{x_{i}} \delta^{\prime}\left(x, x_{i}\right) \sin \frac{n \pi x}{a} \sin \frac{m \pi y}{b} d x d y \\
& \quad=G_{i} K_{i}\left(\frac{m \pi}{a}\right) \cos \frac{m \pi x_{i}}{a}\left(\frac{n \pi}{b}\right)^{2} \int_{0}^{b}\left(\frac{\partial w}{\partial x}\right)_{x_{i}} \sin ^{\frac{n \pi}{b}} d y
\end{aligned}
$$

次に更に( 2$) \sim(5)$ 式に変数 $\boldsymbol{y}$ に関してフーリエ変換を適用すると,

$$
\begin{aligned}
& E_{0} I_{0}\left[\left(\frac{n \pi}{b}\right)^{4} \int_{0}^{b} w_{0} \sin \frac{n \pi y}{b} d y+\left(\frac{n \pi}{b}\right)\left\{\left(\frac{\partial^{2} w}{\partial y^{2}}\right)_{0}^{0}-(-1)^{n}\left(\frac{\partial^{2} w}{\partial y^{2}}\right)_{0}^{b}\right\}\right\} \\
& =D \int_{0}^{b}\left\{\left(\frac{\partial^{3} w}{\partial x^{3}}\right)_{0}+(2-\nu)\left(\frac{n \pi}{b}\right)^{2}\left(\frac{\partial w}{\partial x}\right)_{0}\right\} \sin \frac{n \pi y}{b} d y \\
& E_{a} I_{a}\left[\left(\frac{n \pi}{b}\right)^{4} \int_{0}^{b} w_{a} \sin \frac{n \pi y}{b} d y+\left(\frac{n \pi}{b}\right)\left\{\left(\frac{\partial^{2} w}{\partial y^{2}}\right)_{a}^{0}-(-1)^{n}\left(\frac{\partial^{2} w}{\partial y^{2}}\right)_{a}^{b}\right\} !\right. \\
& =D \int_{0}^{b}\left\{\left(\frac{\partial^{3} w}{\partial x^{3}}\right)_{a}+(2-v)\left(\frac{n \pi}{b}\right)^{2}\left(\frac{\partial w}{\partial x}\right)_{a}\right\} \sin \frac{n \pi y}{b} \alpha y \\
& -G_{0} K_{0}\left(\frac{n \pi}{b}\right)^{2} \int_{0}^{b}\left(\frac{\partial w}{\partial x}\right)_{0} \sin \frac{n \pi y}{b} d y=\int_{0}^{l}\left(\frac{\partial^{2} w}{\partial x^{2}}\right)_{0} \sin \frac{n \pi y}{b} d y+v\left(\frac{n \pi}{b}\right)^{2} \int_{0}^{b} w_{0} \sin \frac{n \pi y}{b} d y \\
& -G_{a} K_{a}\left(\frac{n \pi}{b}\right)^{2} \int_{0}^{b}\left(\frac{\partial w}{\partial x}\right)_{a} \sin \frac{n \pi y}{b} d y=\int_{0}^{b}\left(\frac{\partial^{2} w}{\partial x^{2}}\right)_{a} \sin \frac{n \pi y}{b} d y+v\left(\frac{n \pi}{b} \int_{0}^{b} w_{a} \sin \frac{n \pi y}{b} d y\right.
\end{aligned}
$$

(21)〜(24)式に( 6$) \sim(11)$ 式を代入すれば,

$$
\begin{gathered}
E_{i} I_{i} \int_{0}^{a} \int_{0}^{b}\left(\frac{\partial^{4} w}{\partial y^{4}}\right)_{x_{i}} \delta\left(x, x_{i}\right) \sin \frac{m \pi x}{a} \sin \frac{n \pi y}{b} d x d y \\
=E_{i} I_{i}\left(\frac{n \pi}{b}\right)^{4} \sin \frac{m \pi x_{i}}{a} \int_{0}^{b} w_{x_{i}} \sin \frac{n \pi y}{b} d y \\
\quad-\left\{C_{i}^{0}\left(\frac{n \pi}{b}\right)\left(\frac{\partial w}{\partial y}\right)_{i}^{0}-C_{i}^{b}\left(\frac{n \pi}{b}\right)(-1)^{n}\left(\frac{\partial w}{\partial y}\right)_{i}^{b}\right\} \sin \frac{m \pi x_{i}}{a} \\
E_{0} I_{0}\left(\frac{n \pi}{b}\right)^{4} \int_{0}^{b} w_{0} \sin \frac{n \pi y}{b} d y-C_{0}^{0}\left(\frac{n \pi}{b}\right)\left(\frac{\partial w}{\partial y}\right)_{0}^{0}+C_{0}^{b}(-1)^{n}\left(\frac{n \pi}{b}\right)\left(\frac{\partial w}{\partial y}\right)_{0}^{b} \\
\left.=D \int_{0}^{b}\left\{\frac{\partial^{3} w}{\partial x^{3}}\right)_{0}+(2-v)\left(\frac{n \pi}{b}\right)^{2}\left(\frac{\partial w}{\partial x}\right)_{0}\right\} \sin \frac{n \pi y}{b} d y \\
\left.E_{a} I_{a}\left(\frac{n \pi}{b}\right)^{4} \int_{0}^{b} w_{a} \sin \frac{n \pi y}{b} d y-C_{a}^{0^{\prime}} \frac{n \pi}{b}\right)\left(\frac{\partial w}{\partial x}\right)_{a}^{0}+C_{a}^{b}(-1)^{n}\left(-\frac{n \pi}{b}\right)\left(\frac{\partial w}{\partial y}\right)_{a}^{b} \\
=D \int_{0}^{b}\left\{\left(\frac{\partial^{3} w}{\partial x^{3}}\right)_{a}+(2-v)\left(\frac{n \pi}{b}\right)^{2}\left(\frac{\partial w}{\partial x}\right)_{a}\right\} \sin \frac{n \pi y}{b} d y
\end{gathered}
$$


此処で(1)，(22)，(25)，(25)，(26)〜(29)及(20)式を(19)式に入れると，

$$
\begin{aligned}
& \int_{0}^{a} \int_{0}^{b} w \sin \frac{m \pi x}{a} \sin \frac{n \pi y}{b} d x d y \\
& =-\frac{1}{D\left\{\left(\frac{m \pi}{a}\right)^{2}+\left(\frac{n \pi}{b}\right)^{2}\right\}^{2}} \int_{0}^{a} \int_{0}^{b} p \sin \frac{m \pi x}{a} \sin \frac{n \pi y}{b} d x d y \\
& -\sum_{i} \frac{E_{i} I_{i}}{D} \frac{\left(\frac{n \pi}{b}\right)^{4} \sin \frac{m \pi x_{i}}{a}}{\left\{\left(\frac{m \pi}{a}\right)^{2}+\left(\frac{n \pi}{b}\right)^{2}\right\}^{2}} \int_{0}^{D} w_{x_{i}} \sin \frac{n \pi y}{b} d y \\
& +\sum_{i} \frac{G_{i} K_{i}}{D} \frac{\left(\frac{m \pi}{a}\right)\left(\frac{n \pi}{b}\right)^{2} \cos \frac{m \pi x_{i}}{a}}{\left\{\left(\frac{m \pi}{a}\right)^{2}+\left(\begin{array}{c}
n \pi \\
b
\end{array}\right)^{2}\right\}^{2}} \int_{0}^{b}\left(\frac{\partial w}{\partial x}\right)_{i} \sin \frac{n \pi y}{b} d y \\
& +\sum_{i} \frac{C_{i}^{0} i}{D} \frac{\left(\begin{array}{c}
n \pi \\
b
\end{array}\right) \sin \frac{m \pi x_{i}}{a}}{\left\{\left(\frac{m \pi}{a}\right)^{2}+\left(\frac{n \pi}{b}{ }^{2}\right\}^{2}\right.}\left(\frac{\partial w}{\partial y}\right)_{i}^{0}-\sum_{i} \frac{C_{i}^{b}\left(\begin{array}{c}
n \pi \\
b
\end{array}\right)(-1)^{n} \sin \frac{m \pi x_{i}}{a}}{\left\{\left(\frac{m \pi}{a}\right)^{2}+\left(\frac{n \pi}{b}\right)^{2}\right\}^{2}}\left(\frac{\partial w}{\partial y}\right)^{b} \\
& +\frac{G_{0} K_{0}}{D} \frac{\left(\frac{m \pi}{a}\right)\left(\begin{array}{c}
n \pi \\
b
\end{array}\right)^{2}}{\left\{\left(\frac{m \pi}{a}\right)^{2}+\left(\frac{n \pi}{b}\right)^{2}\right\}^{2}} \int_{0}^{b}\left(\frac{\partial w}{\partial x}\right)_{0} \sin \frac{n \pi y}{b} d y \\
& -\frac{G_{a} K_{a}}{D} \frac{(-1) m\left(\frac{m \pi}{a}\right)\left(\frac{n \pi}{b}\right)^{2}}{\left\{\left(\frac{m \pi}{a}\right)^{2}+\left(\frac{n \pi}{b}\right)^{2}\right\}^{2}} \int_{0}^{b}\left(\frac{\partial w}{\partial x}\right)_{a} \sin \frac{n \pi y}{b} d y \\
& -\frac{\left(\frac{m \pi}{a}\right)^{3}+(2-v)\left(\frac{m \pi}{a}\right)\left(\frac{n \pi}{b}\right)^{2}}{\left\{\left(\frac{m \pi}{a}\right)^{2}+\left(\frac{n \pi}{b}\right)^{2}\right\}^{2}}\left[\frac{D}{E_{0} I_{0}}-\frac{1}{\left(\frac{n \pi}{b}\right)^{4}} \int_{0}^{b}\left\{\left(\frac{\partial^{3} w}{\partial x^{3}}\right)_{0}\right.\right. \\
& \left.\left.+(2-v)\left(\begin{array}{c}
n \pi \\
b
\end{array}\right)^{2}\left(\frac{\partial w}{\partial x}\right)_{0}\right\} \sin \frac{n \pi y}{b} d y+\frac{C_{0}^{0}}{E_{0} I_{0}} \frac{1}{\left(\begin{array}{c}
n \pi \\
b
\end{array}\right)^{3}}\left(\frac{\partial w}{\partial y}\right)_{0}^{0}-\frac{C_{0}^{b}}{E_{0} I_{0}} \frac{1}{\left(\frac{n \pi}{b}\right)^{3}}\left(\frac{\partial w}{\partial y}\right)_{0}^{b}\right] \\
& +\frac{\left(\frac{m \pi}{a}\right)^{3}+(2-v)\left(\frac{m \pi}{a}\right)\left(\frac{n \pi}{b}\right)^{2}}{\left\{\left(-\frac{m \pi}{a}\right)^{2}+\left(\frac{n \pi}{b}\right)^{2}\right\}^{2}}(-1)^{\frac{m}{r}}-\frac{D}{E_{a} I_{a}} \frac{1}{\left(\frac{n \pi}{b}\right)^{4}} \int_{0}^{b}\left\{\left(\frac{\partial^{3} w}{\partial x^{3}}\right)_{a}\right. \\
& \left.+(2-v)\left(\frac{n \pi}{b}\right)^{2}\left(\frac{\partial w}{\partial x}\right)_{a}\right\} \sin \frac{n \pi y}{b} d y+\frac{C_{a}^{a}}{E_{a} I_{a}} \frac{1}{\left(\frac{n \pi}{b}\right)^{3}}\left(\frac{\partial w}{\partial y}\right)_{a}^{0}-\frac{C_{a}^{b}}{E_{a} I_{a}} \frac{1}{\left(\frac{n \pi}{b}\right)^{3}}\left(\frac{\partial w}{\partial y}\right)_{a}^{b}
\end{aligned}
$$

我々はここで(30)式の両辺にフーリエの逆変換を施す。先ず $m$ について逆変掺を行う。

$$
\begin{aligned}
& \int_{0}^{b} w \sin \frac{n \pi y}{b} d y=\frac{2}{D a} \sum_{m} \frac{\sin ^{-\frac{m \pi x}{a}}}{\left\{\left(\frac{m \pi}{a}\right)^{2}+\left(\begin{array}{c}
n \pi \\
b
\end{array}\right)^{2}\right\}^{2}} \int_{0}^{a} \int_{0}^{b} p \sin \frac{m \pi \xi}{a} \sin \frac{n \pi \eta}{b} d \xi d \eta \\
& -\sum_{i} \sum_{m} \frac{2 E_{i} I_{i}}{D a} \frac{\left(\frac{n \pi}{b}\right)^{4} \sin \frac{m \pi x_{i}}{a} \sin \frac{m \pi x}{a}}{\left\{\left(\frac{m \pi}{a}\right)^{2}+\left(\begin{array}{c}
n \pi \\
b
\end{array}\right)^{2}\right\}^{2}} \int_{0}^{b} w_{x_{i}} \sin \frac{n \pi y}{b} d y \\
& +\sum_{i} \sum_{m} \frac{2 G_{i} K_{i}}{D a} \frac{\left(\frac{m \pi}{a}\right)\left(\frac{n \pi}{b}\right)^{2} \cos \frac{m \pi x_{i}}{a} \sin \frac{m \pi x}{a}}{\left\{\left(\frac{m \pi}{a}\right)^{2}+\left(\frac{n \pi}{b}\right)^{2}\right\}^{2}} \int_{0}^{b}\left(\frac{\partial w}{\partial x}\right)_{x i} \sin ^{\frac{n \pi y}{b}-d y}
\end{aligned}
$$




$$
\begin{aligned}
& +\sum_{i} \sum_{m} \frac{2 C_{i}^{0}}{D a} \frac{\left(\frac{n \pi}{b}\right) \sin \frac{m \pi x_{i}}{a} \sin \frac{m \pi x}{a}}{\left\{\left(\frac{m \pi}{a}\right)^{2}+\left(\frac{n \pi}{b}\right)^{2}\right\}^{2}}\left(\frac{\partial w}{\partial y}\right)_{x_{i}}^{0} \\
& -\sum_{i} \sum_{m} \frac{2 C_{i}^{b}}{D a} \frac{(-1)^{n}\left(\frac{n \pi}{b}\right) \sin \frac{m \pi x_{i}}{a} \sin \frac{m \pi x}{a}}{\left\{\left(\frac{m \pi}{a}\right)^{2}+\left(\frac{n \pi}{b}\right)^{2}\right\}^{2}}\left(\frac{\partial w}{\partial y}\right)_{x_{i}}^{b} \\
& +\frac{2 G_{0} K_{0}}{D a} \sum_{m} \frac{\left.\left(\frac{m \pi}{a}\right) \frac{n \pi}{b}\right)^{2} \cdot \sin \frac{m \pi x}{a}}{\left\{\left(\frac{m \pi}{a}\right)^{2}+\left(\frac{n \pi}{b}\right)^{2}\right\}^{2}} \int_{0}^{3}\left(\frac{\partial w}{\partial x}\right)_{0} \sin \frac{n \pi y}{b} d y \\
& -\frac{2 G_{a} K_{a}}{D a} \sum_{m} \frac{(-1)^{n}\left(\frac{m \pi}{a}\right)\left(\frac{n \pi}{b}\right)^{2} \sin ^{\frac{m \pi x}{x}}}{\left\{\left(\frac{m \pi}{a}\right)^{2}+\left(\begin{array}{c}
n \pi \\
b
\end{array}\right)^{2}\right\}^{2}} \int_{0}^{3}\left(\frac{\partial w}{\partial x}\right)_{a} \sin \frac{n \pi y}{b} d y \\
& -\sum_{m} \frac{\left(\frac{m \pi}{a}\right)^{3}+(2-v)\left(\frac{m \pi}{a}\right)\left(-\frac{n \pi}{b}\right)^{2}}{\left\{\left(\frac{m \pi}{a}\right)^{2}+\left(\frac{n \pi}{b}\right)^{2}\right\}^{2}} \sin \frac{m \pi x}{a}<\frac{2 D}{a E_{0} I_{0}} \frac{1}{\left(\frac{n \pi}{b}\right)^{4}} \int_{0}^{b}\left\{\frac{\partial^{3} w}{\partial x^{3}}\right)_{0} \\
& \left.+(2-v)\left(\frac{n \pi}{b}\right)^{2}\left(\frac{\partial w}{\partial x}\right)_{0}\right\} \sin \frac{n \pi y}{b} d y \\
& \left.+\frac{2 C_{0}^{0}}{E_{0} I_{0} a} \frac{1}{\left(\frac{n \pi}{b}\right)^{3}}\left(\frac{\partial w}{\partial y}\right)_{0}^{0}-\frac{2 C_{0}^{b}}{E_{0} I_{0} a} \frac{(-1)^{n}}{\left(\frac{n \pi}{b}\right)^{3}}\left(\frac{\partial w}{\partial y}\right)_{0}^{b}\right] \\
& +\sum_{m} \frac{\left(\frac{m \pi}{a}\right)^{3}+(2-v)\left(\frac{m \pi}{a}\right)\left(\frac{n \pi}{b}\right)^{2}}{\left\{\left(-\frac{m \pi}{a}\right)^{2}+\left(-\frac{n \pi}{a}\right)^{2}\right\}^{2}}(-1)^{9 r} \sin \frac{m \pi x}{a}\left[\frac{2 D}{a E_{0} I_{0}} \frac{1}{\left(\frac{n \pi}{b}\right)^{4}}\right. \\
& \times \int_{0}^{\dot{b}}\left\{\left(\frac{\partial^{3} w}{\partial x^{3}}\right)_{a}+(2-v)\left(\frac{n \pi}{b}\right)^{2}\left(\frac{\partial w}{\partial x}\right)_{0}^{i} \sin \frac{n \pi y}{b} d y\right. \\
& \left.+\frac{2 C_{a}^{0}}{E_{a} I_{a} a} \frac{1}{\left(\frac{n \pi}{b}\right)^{3}}\left(\frac{\partial w}{\partial y}\right)_{a}^{0}-\frac{2 C_{a}^{b}}{E_{a} I_{a}} \frac{(-1)^{n}}{\left(\frac{n \pi}{b}\right)^{3}}\left(\frac{\partial w}{\partial y}\right)_{a}^{b}\right]
\end{aligned}
$$

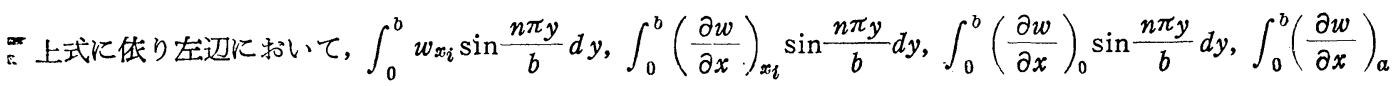

$$
\times \sin \frac{n \pi y}{b} d y, \int_{0}^{b}\left\{\left(\frac{\partial^{3} w}{\partial x^{3}}\right)_{0}+(2-v)\left(\frac{n \pi}{b}\right)^{2}\left(\frac{\partial w}{\partial x}\right)_{0}\right\} \sin \frac{n \pi y}{b} d y \text { 及ひ" }
$$

$\int_{0}^{b}\left\{\left(\frac{\partial^{3} w}{\partial x^{3}}\right)_{a}+(2-v)\left(-\frac{n \pi}{b}\right)^{2}\left(\frac{\partial w}{\partial x}\right)_{a}\right\} \sin \frac{n \pi y}{b} d y$ を作ると,これ等を変数とする $z i+4$ 元の連立方程式が出 来る。但しこの場合 $\left(\frac{\partial w}{\partial y}\right)_{x_{i}}^{0},\left(\frac{\partial w}{\partial y}\right)_{x_{i}}^{b},\left(\frac{\partial w}{\partial y}\right)_{0}^{0},\left(\frac{\partial w}{\partial y}\right)_{0}^{b},\left(\frac{\partial w}{\partial y}\right)_{a}^{0},\left(\frac{\partial w}{\partial y}\right)_{a}^{b}$ は一心既知数と見做すことに する。この連立方程式を仮りに(32)式とすれば, (32)式を解いて变数を決定し,これ等を(31)式に代入して新たに これを(33(式と命名する。ここにおいて(33)式の両辺に $n$ について逆フーリ土変换を施すと， wが得られる， これを(34)式とする。この(34)式の右辺には前述の $\left(\frac{\partial w}{\partial y}\right)_{x i}^{0}, \cdots か ゙$ 未知項として含まれているのでこれ等を決定 しなければならない。

先ず(34)式の左辺に执いて前と同様にして, $\left(\frac{\partial w}{\partial y}\right)_{x_{i},}^{0}\left(\frac{\partial w}{\partial y}\right)_{x_{i},}^{b}\left(\frac{\partial w}{\partial y}\right)_{0}^{0},\left(\frac{\partial w}{\partial y}\right)_{0,}^{b},\left(\frac{\partial w}{\partial y}\right)_{a}^{0},\left(\frac{\partial w}{\partial y}\right)_{a}^{b}$ を 
作ると, これ等を変数とする $2 i+4$ 元の連立方程式が得られる。これを(35)式とすれば, (35)式を解いて変数を 決定し之等を(34)式に代入して新たにこれを（36）式と命名すれば，(36)式は(18)式の解として求められたわけ である。

\section{§5 無限長板に一定の間隔をおいて防撓材が附せられている場合}

この場合には境界条件として下記の如く，実用化を考慮して簡略化する。

即ち力学諸状態は第 2 図に示された各パネル 每全く同様であつて全体としては一つのパネル 間の繰返しであると仮定する。かく仮定すれば 我々は或る一つのパネルについて論ずればよい わけであつて, 以下に於いてはこの一つのパネ ルを取扱ら。従つてもはやこのパネルには防挩 材は辺にのみ附されているだけで内部には附さ れていない。依つて我々は先ず $x=0, a$ の辺が 防撓材に上り支持され，乙かもこの防撓材の両 端には封板が固着されているものとし， $y=0, b$
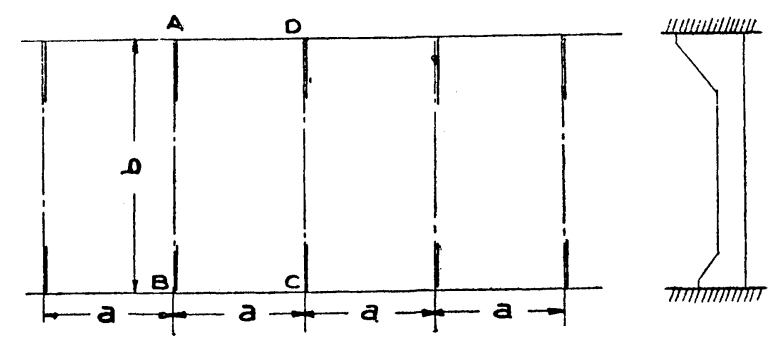

Fig. 2

の辺は肘板のある点を除き緢純支持と仮定する。

更に荷重並びに撓等の諸量すべはて $x=a / 2$ に対して対称であると仮定する。斯様な仮定の下に(31)式は下 記の形になる。

$$
\begin{aligned}
& \int_{0}^{b} w \sin \frac{n \pi y}{b} d y=\frac{4}{D a} \sum_{m}\left\{\left(\frac{m \pi}{a}\right)^{2}+\left(\frac{n \pi}{b}\right)^{2}\right\}^{2} \int_{0}^{b} \int_{0}^{a} p \sin \frac{m \pi \xi}{a} \sin \frac{n \pi \eta}{b} d \xi d \eta \\
& +\frac{4 G_{0} K_{0}}{D a} \sum_{m} \frac{\left(\frac{m \pi}{a}\right)\left(\frac{n \pi}{b}\right)^{2} \sin \frac{m \pi x}{a}}{\left\{\left(\frac{m \pi}{a}\right)^{2}+\left(\frac{n \pi}{b}\right)^{2}\right\}^{2}} \int_{0}^{b}\left(\frac{\partial w}{\partial x}\right)_{0} \sin \frac{n \pi y}{b} d y \\
& +\sum_{m} \frac{\left(\frac{m \pi}{a}\right)^{3}+(2-v)\left(\frac{m \pi}{a}\right)\left(\frac{n \pi}{b}\right)^{2}}{\left\{\left(\frac{m \pi}{a}\right)^{2}+\left(\frac{n \pi}{b}\right)^{2}\right\}^{2}} \sin \frac{m \pi x}{a}\left[\frac { 4 D } { a E _ { 0 } I _ { 0 } } \frac { 1 } { ( \frac { n \pi } { b } ) ^ { 4 } } \int _ { 0 } ^ { b } \left\{\left(\frac{\partial^{3} w}{\partial x^{3}}\right)_{0}\right.\right. \\
& \left.+(2-v)\left(\begin{array}{c}
n \pi \\
b
\end{array}\right)^{2}\left(\frac{\partial w}{\partial x}\right)_{0}\right\} \sin \frac{n \pi y}{b} d y \\
& \left.+\frac{4 C_{0}^{0}}{E_{0} I_{0} a} \frac{1}{\left(-\frac{n \pi}{b}\right)^{3}}\left(\frac{\partial w}{\partial y}\right)_{0}^{0}-\frac{4 C_{0}^{b} .}{E_{0} I_{0} a} \frac{(-1)^{n}}{\left(\frac{n \pi}{b}\right)^{3}}\left(\frac{\partial w}{\partial y}\right)_{0}^{b}\right]
\end{aligned}
$$

但しここで $m$ は奇数とする。

〔1〕 $C_{0}^{0}=C_{0}^{b}=0$ の場合

(37)式を一般理論で述べた方法で解くと， $w=\sum_{m, n} a_{m n n} \sin \frac{m \pi x}{a} \sin \frac{n \pi y}{a}$ の倸数 $a_{m n n}$ は次の如くなる。

$$
\begin{gathered}
a_{m n}=a^{0} m_{m b}+\frac{m^{3}+(2-v) m \lambda^{2} n^{2}}{\left(m^{2}+\lambda^{2} n^{2}\right)^{2}} \frac{\left(1+\theta_{0} A\right) G+\theta_{0}(\nu A+B) F}{K\left(1+\theta_{0} A\right) \lambda^{2} n^{2}+\theta_{0}(A+B)^{2}+\left(1-\nu^{2}\right) A+2(1-\nu) B} \\
-\frac{\theta_{0} m \lambda^{2} n^{2}}{\left(m^{2}+\lambda^{2} n^{2}\right)^{2}} \frac{\left\{K \lambda^{2} n^{2}+\left(1-\nu^{2}\right) A+2(1-\nu) B\right\} F-(\nu A+B) G}{K\left(1+\theta_{0} A\right) \lambda^{2} n^{2}+\theta_{0}(A+B)^{2}+\left(1-\nu^{2}\right) A+2(1-\nu) B}
\end{gathered}
$$

ここで $\theta_{0}=\infty$ とすると，

$$
a_{m n}=a_{m n}^{0}+\frac{m^{3}+2 m \lambda^{2} n^{2}}{\left(m^{2}+\lambda^{2} n^{2}\right)^{3}} H-\frac{m \lambda^{2} n^{2}}{\left(m^{2}+\lambda^{2} n^{2}\right)} \frac{1}{A}(F-B H)
$$

$\theta_{\theta}=0$ とすると, 


$$
a_{m n}=a_{2 n n}^{o}+\frac{G}{K \lambda^{2} n^{2}+\left(1-v^{2}\right) A+2(1-v) B} \frac{m^{3}+(2-v) m \lambda^{2} n^{2}}{\left(m^{2}+\lambda^{2} n^{2}\right)^{2}}
$$

(38)〜 (40) は一般的な荷重即ち分布荷重並に集中荷重の場合であるが, 今特に $x=0, a$ の防撓材上に $p う(x, 0)$. $p \delta(x, a)$ なる集中荷重が作用し，その外には何ら荷重が作用しない場合には,

$$
\begin{aligned}
a_{i n: 2} & =\frac{2 a^{2}}{\pi^{3} D \lambda n^{2}} \frac{m^{3}+(2-v) m \lambda^{2} n^{2}}{\left(m^{2}+\lambda^{2} n^{2}\right)^{2}} \quad K\left(1+\theta_{0} A\right) \lambda^{2} n^{2}+\theta_{0}(A+B)^{2}+\left(1-v^{2}\right) A+2(1-v) B \\
& +\frac{2 a^{2}}{\pi^{3} D \lambda n^{2}} \frac{\theta_{0} m \lambda^{2} n^{2}}{\left(m^{2}+\lambda^{2} n^{2}\right)^{2}}-\frac{v A+B}{K\left(1+\theta_{0} A\right) \lambda^{2} n^{2}+\theta_{0}(A+B)^{2}+\left(1-v^{2}\right) A+2(1-v) B} p_{c}^{0}
\end{aligned}
$$

ここで $\theta_{0}=\infty$ とすると,

$$
\begin{aligned}
a_{m n n} & =\frac{2 a^{2}}{\pi^{3} D \lambda n^{2}}\left\{\frac{m^{3}+2 m \lambda^{2} n^{2}}{\left(m^{2}+\lambda^{2} n^{2}\right)^{2}} \frac{A}{K A \lambda^{2} n^{2}+(A+B)^{2}} p_{c}^{0}\right. \\
& \left.+\frac{m \lambda^{2} n^{2}}{\left(m^{2}+\lambda^{2} n^{2}\right)^{2}} \frac{B}{K A \lambda^{2} n^{2}+(A+B)^{2}} p_{c}^{0}\right\}
\end{aligned}
$$

$\theta_{0}=0$ とすると

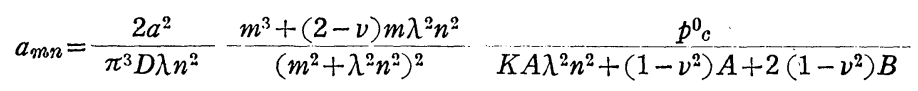

[2] $C_{0}^{0} \neq 0, C_{0}^{b} \neq 0$ の場合

$$
\theta_{0}=\infty \text { とすると }
$$

$$
\begin{aligned}
a_{m n}= & a_{m n}^{\prime}-\frac{K A}{K A \lambda^{2} n^{2}+(A+B)^{2}} \frac{1}{\lambda n}\left\{U-(-1)^{n} V\right\} \\
& \left\{\begin{array}{l}
m^{3}+2 m \lambda^{2} n^{2} \\
\left.\left(m^{2}+\lambda^{2} n^{2}\right)^{2}+\frac{B}{A}-\frac{m \lambda^{2} n^{2}}{\left(m^{2}+\lambda^{2} n^{2}\right)^{2}}\right\}
\end{array}\right.
\end{aligned}
$$

但しここで $a_{m n n}^{\prime}=a_{m n}^{0}+\frac{m^{3}+2 n \lambda^{2} n^{2}}{\left(m^{2}+\lambda^{2} n^{2}\right)^{2}} H-\frac{m \lambda^{2} n^{2}}{\left(m^{2}+\lambda^{2} n^{2}\right)^{2}} \frac{1}{A}(F-B H)$

$$
\begin{aligned}
& U=\frac{\bar{C}_{0}^{0} N_{1}+\bar{C}_{0}^{0} \bar{C}_{0}^{b}\left(M_{1} N_{1}-M_{2} N_{2}\right)}{1+\bar{C}_{0}^{0} M_{1}+\bar{C}_{0}^{b} M_{1}+\bar{C}_{0}^{0} \bar{C}_{0}^{b}\left(M_{1}^{2}+M_{2}^{2}\right)} \\
& V=\frac{\bar{C}_{0}^{b} N_{2}+\bar{C}_{0}^{0} \bar{C}_{0}^{b}\left(M_{2} N_{1}-M_{1} N_{2}\right)}{1+\bar{C}_{0}^{0} M_{1}+\bar{C}_{0}^{b} M_{1}+\bar{C}_{0}^{0} \bar{C}_{b}^{\mathrm{b}}\left(M_{1}^{2}+M_{2}^{2}\right)}
\end{aligned}
$$

(a) $C_{0}^{0}=C_{0}^{b}=C$ なる時

同時に荷重が $y=b / 2$ に対して対称であるとすると,

$$
a_{i \omega n}=a_{m n}^{\prime}-\frac{K A}{K A \lambda^{2} n^{2}+(A+B)^{2}} \frac{U}{\lambda n}\left\{-\frac{m^{3}+2 m \lambda^{2} n^{2}}{\left(m^{2}+\lambda^{2} n^{2}\right)^{2}}+\frac{B}{A} \frac{m \lambda^{2} n^{2}}{\left(m^{2}+\lambda^{2} n^{2}\right)^{2}}\right\}
$$

但乙 $U=\frac{2 \bar{C} N_{1}{ }^{\prime}}{1+2 C} M_{1}{ }^{\prime}, m, n$ は奇数, $\bar{C}=\bar{C}_{0}^{0}=\bar{C}_{0}^{b}$

ここで $C=\infty$ とすると, (45)式において $U=\frac{N_{1}^{\prime}}{M_{1}^{\prime}}$

(b) $C_{0}^{0} \neq 0, C_{o}^{\mathrm{b}}=0$ なる時

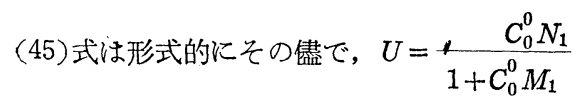

此处で $C_{0}^{0}=\infty$ とすると $U=\frac{N_{1}}{M_{1}}$

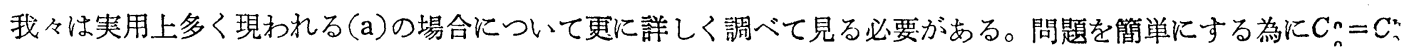
$=\infty$ とし, 荷重は $x=a / 2$ に対して対称又 $y=b / 2$ に対しても同様に対称とすると,(45)式は次の如く畫き直さ 
れる。

$$
\begin{gathered}
a_{m n n}=a_{m n}^{0}-\frac{m \lambda^{2} n^{2}}{\left(m^{2}+\lambda^{2} n^{2}\right)^{2}} \frac{E}{A}+\left(H-\frac{N_{1}^{\prime}}{M_{1}^{\prime}} \frac{K A}{K A \lambda^{2} n^{2}+(A+B)^{2}} \frac{1}{\lambda n}\right) \\
\left\{\frac{1}{m}-\frac{\lambda^{4} n^{4}}{m\left(m^{2}+\lambda^{2} n^{2}\right)^{2}}+\frac{B}{A}-\frac{m \lambda^{2} n^{2}}{\left(m^{2}+\lambda^{2} n^{2}\right)^{2}}\right\}
\end{gathered}
$$

$x=0$ における撓, 板に生ずる曲げモーメント，防撓材に生ずる曲げモーメント，を夫々下記の形式で表わする のとすると，

$$
\begin{aligned}
& w=\sum_{n} a_{n} \sin \frac{n \pi y}{b}, \quad M_{x}=\sum_{n} s_{n} \sin \frac{n \pi y}{b}, M_{y}=\sum_{n} t_{n} \sin \frac{n \pi y}{b} \\
& M_{s}=\sum_{n} q_{n} \sin \frac{n \pi y}{b} \\
& a_{n}=\pi / 4\left\{H-\frac{1}{\lambda n}-\frac{N_{1^{\prime}}}{M_{1^{\prime}}} \frac{K A}{K A \lambda^{2} n^{2}+(A+B)}\right\} \\
& s_{n}=\frac{\pi^{3} D}{4 a^{2}} \frac{\lambda^{2} n^{2}}{K A \lambda^{2} n^{2}+(A+B)^{2}}\left[\left\{K \lambda^{2} n^{2}+A+(2-v) B\right\} F-(v A+B) G_{1}+\frac{N_{1^{\prime}}}{M_{1^{\prime}}} \frac{K}{\lambda n}(v A+B)\right] \\
& t_{n}=\frac{\pi^{3} D}{4 a^{2}} \frac{\lambda^{2} n^{2}}{K A \lambda^{2} n^{2}+(A+B)^{2}}\left[\left\{\nu K \lambda^{2} n^{2}+\nu A+(2 \nu-1) B\right\} F-(A+\nu B) G_{1}+\frac{N_{1^{\prime}}}{M_{1^{\prime}}} \frac{K}{\lambda n}(A+\nu B)\right] \\
& q_{n}=\frac{\pi D}{a} \frac{K \lambda^{2} n^{2}}{K A \lambda^{2} n^{2}+(A+B)^{2}}\left\{A G+B F-\frac{N_{1}{ }^{\prime}}{M_{1}{ }^{\prime}} \frac{K}{\lambda n} A\right\}
\end{aligned}
$$

更に防撓材の端部にある肘板に生ずる曲げモーメントを $M_{s}^{0}$ とすると $M_{s}^{0}=\frac{\pi^{2} D}{4 a}-\frac{N_{1}^{\prime}}{M_{1}^{\prime}} K$

以下に集中荷重と一様分布荷重に就いて実例を示す。

1）防撓材上に一椂集中荷重の存在する場合

荷重: $p(y)\{\delta(x, 0)+\delta(x, a)\}$ とする。

$$
\begin{aligned}
& \text { この場合 } a^{0} m n=\frac{8 \lambda a^{2} p_{c}^{0}}{D \pi^{4}} \frac{1}{\left(m^{2}+\lambda^{2} n^{2}\right)^{2}} \sin \frac{m \pi 0}{a} \\
& F=0, \quad G_{1}=\frac{2 a^{2} p_{c}^{0}}{\lambda n^{2} D \pi^{3}}, \quad H=\frac{2 a^{2} p_{c}^{0}}{\lambda n^{2} D \pi^{3}} \frac{A}{K A \lambda^{2} n^{2}+(A+B)^{2}} \\
& N_{1}=\frac{2 a^{2}}{D \pi^{3}} \sum_{\beta} \frac{A p_{c}^{0}}{K A \lambda^{2} n^{2}+(A+B)^{2}} \frac{1}{\beta}, N_{2}=-\frac{2 a^{2}}{D \pi^{3}} \sum_{\beta} \frac{A p_{c}^{0}}{K A \lambda^{2} n^{2}+(A+B)^{2}} \frac{(-1)^{\beta}}{\beta}
\end{aligned}
$$

更に $p_{c}^{0}=\frac{2 a}{\pi \lambda n} p$ の時即ち $y$ 方向に対して荷重が一定なる場合には, 下記の如くなる。以下で $n, \beta$ は奇数と する。

$$
a^{0} m n=\frac{16 a^{3} p}{D \pi^{5}} \frac{1}{n\left(m^{2}+\lambda^{2} n^{2}\right)^{2}} \sin \frac{m \pi 0}{a}, F=0, G=\frac{4 a^{3} p}{D \pi^{4} \lambda^{2} n^{3}}
$$

$H=\frac{4 a^{3} p}{D \pi^{4} \lambda^{2} n^{3}} \frac{A}{K A \lambda^{2} n^{2}+(A+B)^{2}}, \quad N_{1}^{\prime}=-\frac{4 a^{3} p}{D \pi^{4} \lambda} \sum_{\beta} \frac{A}{K A \lambda^{2} \beta^{2}+(A+B)^{2}} \frac{1}{\beta^{2}} \beta$ は奇数ここで特に $\quad N_{c}=\sum_{\beta}$ $\frac{A}{K A \lambda^{2} \beta^{2}+(A+B)^{2}} \frac{1}{\lambda^{2} \beta^{2}}$ と置くと，（ $\beta$ は奇数）

$$
\begin{aligned}
& a_{n}=\frac{a^{3} p \lambda}{D \pi^{3} \lambda n} \frac{A}{K A \lambda^{2} n^{2}+(A+B)^{2}}\left(\frac{1}{\lambda^{2} n^{2}}-\frac{N_{c}}{M}\right) \\
& s_{n}=\frac{a p}{\pi} \lambda^{2} n \frac{\nu A+B}{K A \lambda^{2} n^{2}+(A+B)^{2}}\left(\frac{1}{\lambda^{2} n^{2}}-\frac{N_{c}}{M}\right) \\
& t_{n}=\frac{a p}{\pi}-\lambda^{2} n \frac{A+\nu B}{K A \lambda^{2} n^{2}+(A+B)^{2}}\left(\frac{1}{\lambda^{2} n^{2}}-\frac{N_{c}}{M}\right) \\
& q_{n}=\frac{4 a^{2} p \lambda}{\pi^{3}} \frac{K \lambda n A}{K A \lambda^{2} n^{2}+(A+B)^{2}}\left(\frac{1}{\lambda^{2} n^{2}}-\frac{N_{c}}{M}\right) \\
& M_{s}^{0}=\frac{a^{2} p \lambda}{\pi^{2}}-\frac{N_{c}}{M}, \quad \underset{\substack{y=0 \\
x=0}}{\left(M_{x}\right.}=\frac{a p \lambda}{4}-\frac{\nu}{K} \cdot \frac{N_{c}}{M},\left(M_{y}\right)_{\substack{y=0 \\
x=0}}=\frac{a p \lambda}{4} \frac{1}{K} \frac{N_{c}}{M}
\end{aligned}
$$




$$
\begin{aligned}
& \left(M_{x}\right)_{x=0, y=b / 2}=\frac{a p}{\pi} \sum_{n} \lambda^{2} n \frac{v A+B}{K A \lambda^{2} n^{2}+(A+B)^{2}}(-1)^{\frac{n-1}{2}}\left(\frac{1}{\lambda^{2} n^{2}}-\frac{N_{c}}{M}\right) \\
& \left(M_{y}\right)_{x=0, y=b / 2}=\frac{a p}{\pi} \sum_{n} \lambda^{2} n \frac{A+v B}{K A \lambda^{2} n^{2}+(A+B)^{2}}(-1)^{\frac{n-1}{2}}\left(\frac{1}{\lambda^{2} n^{2}}-\frac{N_{c}}{M}\right) \\
& \left(M_{s}\right)_{y=b / 2}=\frac{4 a^{2} p \lambda}{\pi^{3}} \sum_{n} \frac{K \lambda n A}{K A \lambda^{2} n^{2}+(A+B)^{2}}(-1)^{\frac{n-1}{2}}\left(\frac{1}{\lambda^{2} n^{2}}-\frac{N_{c}}{M}\right)
\end{aligned}
$$

2）矩形板全面に一様分布荷重を受ける場合

$$
\begin{aligned}
& a_{m n}^{0}=\frac{16 a^{4} p_{0}}{D \pi^{6}} \frac{1}{m n\left(m^{2}+\lambda^{2} n^{2}\right)^{2}}, \quad F=\frac{16 a^{4} p_{0}}{D \pi^{6}} \frac{B}{\lambda^{4} n^{5}}, \quad G=\frac{16 a^{4} p_{0}}{D \pi^{6}}-\frac{1}{\lambda^{4} n^{5}}(A+2 B) \\
& H=\frac{16 a^{4} p_{0}}{D \pi^{6}} \frac{1}{\lambda^{4} n^{5}} \frac{(A+B)^{2}}{K A \lambda^{2} n^{2}+(A+B)^{2}}, \quad N_{1}^{\prime}=\frac{16 a^{4} p_{0}}{D \pi^{6}} \sum_{\beta} \frac{1}{\lambda^{3} \beta^{4}} \frac{(A+B)^{2}}{K A \lambda^{2} n^{2}+(A+B)^{2}} \\
& a_{n}=\frac{4 a^{4} p_{0}}{D \pi^{5}} \frac{1}{n}\left[\left\{\frac{1}{\lambda^{4} n^{4}}-\frac{A}{K A \lambda^{2} n^{2}+(A+B)^{2}}-\frac{\sum_{\beta} \frac{1}{\lambda^{4} \beta^{4}}}{M}\right\}-\frac{K A}{K A \lambda^{2} n^{2}+(A+B)^{2}}\left(\frac{1}{\lambda^{2} n^{2}}-\frac{N_{c}}{M}\right)\right] \\
& q_{n}=\frac{16 a^{3} p_{0}}{\pi^{5}} \lambda^{2} n\left[\left\{\frac{1}{\lambda^{4} n^{4}}-\frac{A}{K A \lambda^{2} n^{2}+(A+B)^{2}} \frac{\sum_{\beta} \frac{1}{\lambda^{4} \beta^{4}}}{M} \int^{-}-\frac{K A}{K A \lambda^{2} n^{2}+(A+B)^{2}}\left(\frac{1}{\lambda^{2} n^{2}}-\frac{N_{c}}{M}\right)\right] K\right. \\
& S_{26}=\frac{4 a^{2} p_{0}}{\pi^{3}} \lambda^{2} n\left[\left\{\frac{v}{\lambda^{4} n^{4}}-\frac{A v+B}{K A \lambda^{2} n^{2}+(A+B)^{2}} \frac{\sum_{\beta} \frac{1}{\lambda^{4} \beta^{4}}}{M}\right\}-\frac{K(A v+B)}{K A \lambda^{2} n^{2}+(A+B)^{2}}\left(\frac{1}{\lambda^{2} n^{2}}-\frac{N_{c}}{M}\right)\right] 1 \\
& t_{n}=\frac{4 a^{2} p_{0}}{\pi^{3}} \lambda^{2} n\left[\left\{\frac{1}{\lambda^{4} n^{4}}-\frac{A+B v}{K A \lambda^{2} n^{2}+(A+B)^{2}} \frac{\sum_{\beta} \frac{1}{\lambda^{4} \beta^{4}}}{M}\right\}-\frac{K(A+B v)}{K A \lambda^{2} n^{2}+(A+B)^{2}}\left(\frac{1}{\lambda^{2} n^{2}}-\frac{N_{c}}{M}\right)\right] \\
& M_{8}^{0}=\frac{4 a^{3} p_{0}}{\dot{\pi}^{4}}\left(\frac{\sum_{\beta} \frac{1}{\lambda^{4} \beta^{4}}}{M}-K_{c}^{N_{c}}\right) \\
& \left(M_{x}\right)_{x=0, y=0}=\frac{4 a^{2} p_{0}}{\pi^{2}} \frac{v}{K}\left(\frac{\sum_{\beta} \frac{1}{\lambda^{4} \beta^{4}}}{M}-\frac{K}{M} N_{c}\right),\left(M_{y}\right)_{x=0, y=0}=\frac{a^{2} p_{0}}{\pi^{2}} \frac{1}{K M}\left(\sum_{\beta} \frac{1}{\lambda^{4} \beta^{4}}-K N_{c}\right) \\
& \left(M_{x}\right)_{x=0, y=b_{i} 2}=\frac{4 a^{2} p_{0}}{\pi^{3}} \sum_{n} \lambda^{2} n\left[\left\{\frac{v}{\lambda^{2} n^{2}}-\frac{A v+B}{K A \lambda^{2} n^{2}+(A+B)^{2}} \cdot \frac{\sum_{\beta} \frac{1}{\lambda^{4} \beta^{4}}}{M}\right\}\right. \\
& \left.-\frac{K(A \nu+B)}{K A \lambda^{2} n^{2}+(A+B)^{2}}\left(\frac{1}{\lambda^{4} n^{4}}-\begin{array}{c}
N_{c} \\
M
\end{array}\right)\right](-1)^{\frac{n-1}{2}} \\
& \left(M_{y}\right)_{x=0, y=b^{\prime} 2}=\frac{4 a^{2} p_{0}}{\pi^{3}} \sum_{n} \lambda^{2} n\left[\left\{\frac{1}{\lambda^{4} n^{4}}-\frac{A+B v}{K A} \frac{\sum_{\beta} \frac{1}{\lambda^{2} n^{2}+(A+B)^{2}}}{M}\right\}\right. \\
& \left.-\frac{K(A+\nu B)}{K A \lambda^{2} n^{2}+(A+B)^{2}}\left(\frac{1}{\lambda^{2} n^{2}}-\frac{N_{c}}{M}\right)\right](-1)^{\frac{n-1}{2}} \\
& \left(M_{s}\right)_{y=b \cdot 2}=\frac{16 a^{3} p^{0} K}{\pi^{5}} \sum_{n} \dot{\lambda}^{2} n\left[\left\{\frac{1}{\lambda^{2} n^{4}}-\frac{A}{K A \lambda^{2} n^{2}+(A+B)^{2}} \frac{\sum_{\beta} \frac{1}{\lambda^{4} \beta^{4}}}{M}\right\}\right. \\
& \left.-\frac{K A}{K A \lambda^{2} n^{2}+(A+B)^{2}}\left(\begin{array}{c}
1 \\
\lambda^{2} n^{2}
\end{array}-\begin{array}{c}
N_{c} \\
M
\end{array}\right)\right](-1)^{\frac{n-1}{2}}
\end{aligned}
$$

上部の計算例を以下に示す。

1）防撓材上にのみ荷重のある場合

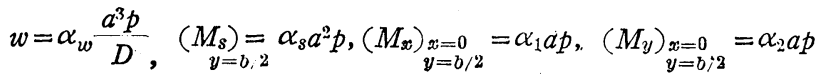

$$
\begin{aligned}
& M_{s}^{0}=\alpha_{s}^{0} a^{2} p,\left(M_{x}\right)_{\substack{x=0 \\
y=0}}=\alpha_{3} a p,\left(M_{y}\right)_{\substack{x=0 \\
b=0}}=\alpha_{4} a p \text { と置き， } \lambda=1 \text { とすれば }
\end{aligned}
$$




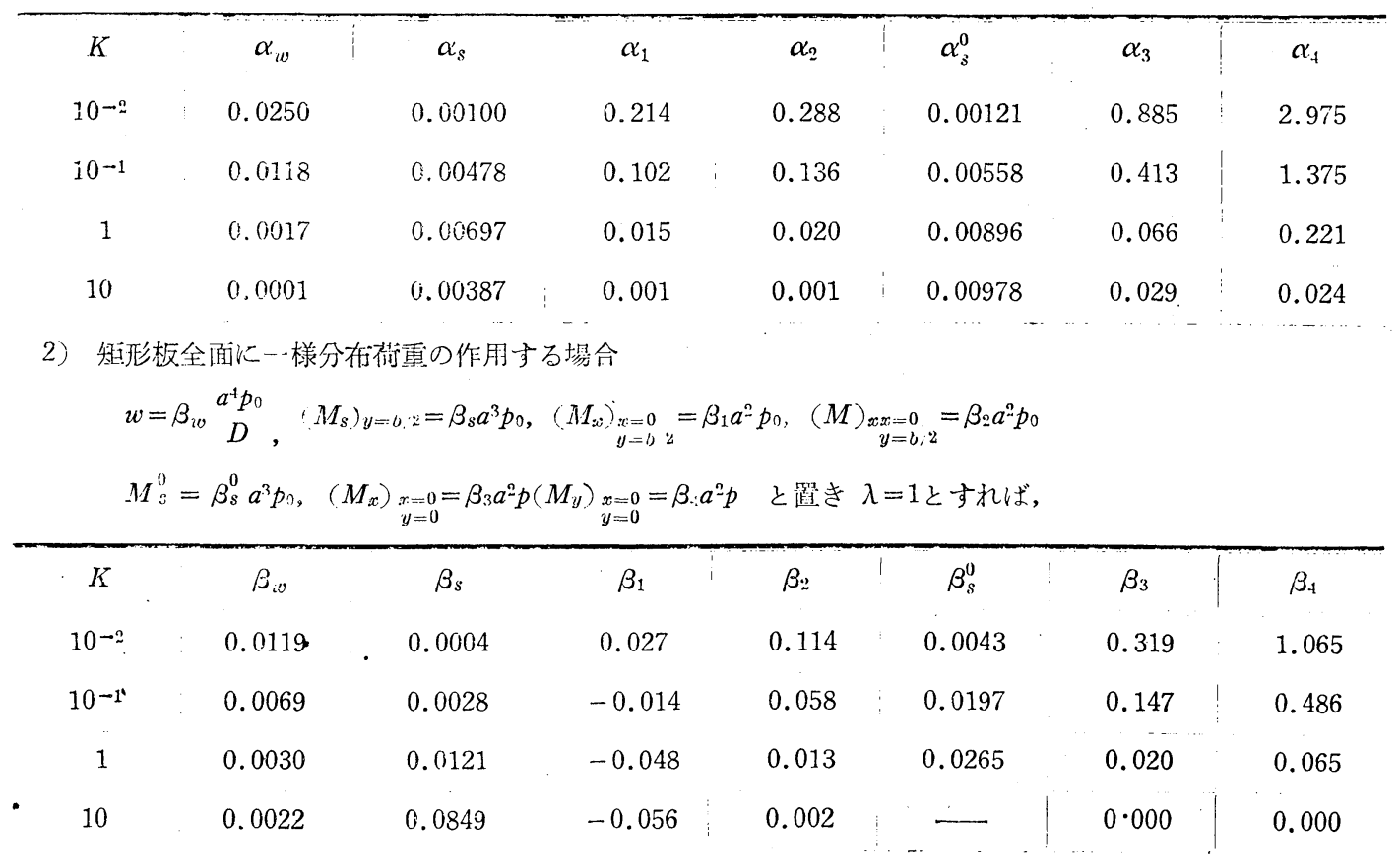

